\title{
A tecnologia e cultura de informação como cenário de pesquisa para a ciência
}

\author{
Greyciane Souza Lins \\ Universidade de Brasília - UnB, Brasil
}

REVIEW

\begin{abstract}
Resumo
Reflete sobre a cultura da informação na internet e suas interpretações científicas. O objetivo é identificar áreas de estudos de cultura da informação do ponto de vista da comunicação e da tecnologia. A partir de uma revisão bibliográfica e descritiva, esboça conceitos de informação e cultura de sob o enfoque de pensamentos históricos e contemporâneos. Conclui que a ciência da informação está implícita nos estudos de cultura da informação por uma perspectiva acadêmica interdisciplinar.
\end{abstract}

Palabras clave

Cultura de informação ; Sociedade da informação ; Tecnologia de informação e comunicação ; Estudos de Internet ; Cibercultura

\section{Technology and information culture as an scenery for research for science}

\begin{abstract}
This paper reflects about the culture of information on the internet and their scientific interpretations. The aim is to identify areas of studies of information culture from the point of view of communication and technology. From a bibliographical and descriptive research, outlines concepts of information and culture from the standpoint of historical and contemporary thoughts. It concludes that the science of information is implicit in the information culture studies by an interdisciplinary academic perspective.
\end{abstract}

Keywords

Information culture; The information society ; Information and communication technology ; Internet studies ; Cyberculture

\section{Introdução}

São visíveis as mudanças ocorridas no cotidiano das pessoas em função de suas interações com as máquinas. Tal evento proporciona facilidades pela técnica e mecanização de processos diários de nossas vidas, e algumas possibilidades tecnológicas podem nos fazer surpreender, pois muitas vezes não conseguimos acompanhar essas mudanças. Atualmente, a sociologia, por exemplo verifica as novas condições que se apresentam na modernidade como consequências das tecnologias de comunicação. Tal análise pode ser observada no olhar de Zygmunt Bauman, em seus estudos sobre o pós-modernismo, ou modernidade liquida. Bauman entende a modernidade como essencialmente, a transformação da sociedade em toda a sua estrutura para uma vida artificial e volátil. Sobre informação, por exemplo, o sociólogo polonês afirma "e aquilo que a informação mais informa é a fluidez do mundo habitado e a flexibilidade dos habitantes” (2001, p.178). As perspectivas discutidas nas ciências que de alguma forma se interessam pela informação ou sobre os seus aspectos levam à elementos que se encaixam em algum tipo de abordagem prática da informação, mesmo que esse não seja o foco ou o objeto principal de investigação científica, como é para a Ciência da Informação. Essa possui a informação como seu principal objeto de pesquisa, e se levarmos em consideração que os sistemas tecnológicos são os principais 
meios de transmissão de informação, se faz pertinente uma averiguação sobre os caminhos que as teorias de estudos de internet, ou seus estudos relacionados como cibercultura e estudos de cultura da informação tratam o objeto informacional.

Portanto, o presente artigo, objetiva demonstrar as possibilidades de investigações científicas sobre a informação no ambiente da tecnologia de redes, cuja principal característica é a rápida mudança do formato de como o fluxo informacional se apresenta. Mostra como outros campos científicos observam a cultura de internet e suas principais interpretações

Essa discussão se faz importante em um momento que existem algumas publicações despontando como literatura de interesse de variados públicos que dizem respeito ao atual estado e ao futuro esperado pela sociedade na era da Internet. Um exemplo é o autor Nicholas Carr, que em seu livro intitulado The shallows: what the internet is doing to our brains (2011), expõe as modificações cognitivas resultantes do uso frequente da web e ilustra, entre outras ocorrências, a dificuldade de concentração que lhe ocorre frequentemente devido à leitura na web: "Quando menciono os meus problemas com leitura para amigos, muitos dizem que estão passando por aflições semelhantes. Quanto mais usam a web, mais têm que se esforçar para permanecer focados em longos trechos de escrita" (p.19). Considerações como essas são pressupostos para o desdobramento das ideias do livro, que possui um sentido não cientifico, mas particular, para associação da comunicação entre usuário e computador, como também ocorre no livro intitulado The glass cage (2014), onde Carr explica a indústria de software no cotidiano e todos os seus reflexos na atenção, memória e felicidade humana.

Vemos não só em Carr, como em Powers (2012) e Johnson (2012), como existe uma necessidade social de responder perguntas sob uma ótica mais próxima do comportamento humano a respeito do que vai acontecer com as nossas próximas gerações, guiadas pelas telecomunicações e excesso de informação. Os Estudos de Internert ou de Cibercultura estão sendo norteados para descrever as possibilidades de interações no póshumanismo nessas questões também.

\section{Quando a tecnologia e a informação se tornam parte da sociedade}

A identificação da relação entre máquinas e seres humanos tem início com o surgimento dos estudos aplicados em Cibernética a partir dos anos 40 do século XX. A ciência de Norbert Wiener observa claramente a simbiose entre o controlador e o controlado ${ }^{1}$, ao interpretar a Cibernética como um sistema que coordena a comunicação, ou como a que máquina controla o fluxo dos sistemas de transformação de informação" (FRANK, 1970, p. 27). Norbert Wiener propusera ao término da II Guerra Mundial o investimento moral e intelectual do Ocidente em uma nova ciência ou forma de pensamento tecnológico, o cibernético, segundo o qual nossos principais problemas sociais e conflitos políticos poderiam ser resolvidos tecnicamente, mediante a sublimação funcional do ser humano em automatismos maquinísticos.

A ideia de um evento ou sistema que funcione a partir de um controle interno, onde a mensagem enviada é recebida e assim trabalhe em harmonia é a mesma utilizada por Claude Shannon e Warren Weaver para compor a teoria matemática da informação. Esta não se preocupa com a mensagem em si, mas com a eficiência no processo de comunicação segundo medidas de informação (SHANNON, 1948; DECHERT, 1970, p. 27).

A principal abordagem da cibernética é o desdobramento que se tem para a aplicabilidade em máquinas e em seres vivos (FRANK, 1970, p 21) e por isso a informação é o seu aspecto central, pois é considerada como o comando que impulsiona o controle de sistemas. Apesar de não ser uma preocupação científica para Wiener, o conceito de informação foi sinteticamente colocado como "o termo que designa o conteúdo daquilo que permutamos com o mundo exterior ao ajustar-nos a ele, e que faz com que nosso ajustamento seja nele percebido" (2006, p. 16-17). A ciência da cibernética entendeu a informação como principal insumo da relação entre a máquina e o homem, conforme verificado por Tofts (2002, p. 3):

A cibernética pressupõe que para entender a natureza humana teve que compreender a informação e ver o ser humano como apenas outro sistema fechado, como uma máquina, adaptando-se e ajustando-se ao seu ambiente em função do controle e fluxo de informações. 
Portanto, o objetivo da cibernética é compreender a sociedade por meio dos processos comunicacionais utilizando-se da informação como a energia que promove seu funcionamento. A percepção é de que sistemas em geral funcionam da mesma maneira, e assim homens e máquinas podem comunicar-se e comunicar entre si.

A cibernética foi vista como uma área cientifica idealizadora de uma sociedade onde as máquinas seriam vistas como parte da coletividade humana. Desde então, essa proposição tem interferido na identidade da informação e da linguagem não só sob a perspectiva da tecnologia como também da sociedade e seus aspectos culturais.

Curiosamente, a precursora sobre os Estudos sobre Cibercultura, Alice Hilton, afirmou em 1964, que a era cibernética seria muito importante para todos por ser uma novidade, e em seu ponto de vista, uma mudança significativa na cultura advinda pelo uso de máquinas inteligentes. Hilton via nesse novo processo, uma ciência, e fundou o Instituto de Pesquisas Ciberculturais, e sugeriu ações para que esse ambiente tecnológico pudesse ser o mais humano possível: políticas governamentais, construções de estruturas urbanas inteligentes, garantia constitucional de qualidade de vida, trabalho para todos, aprendizado continuo, etc (1964, p.150-151). Segundo Rüdiger (2011, p. 8), ela foi a pioneira em usar o termo cibercultura "com o sentido enfático, com uma exigência ética da nova era da automação e das maquinas inteligentes".

Para entender o sentido de Cibercultura, remetemos inicialmente ao seu conceito macro como um contexto social motivado pelo uso de hardware e software com propósito de facilitar o cotidiano humano. Nesse sentido, os elementos de tecnologia (neste caso de informação e comunicação), hardware e software, estão atualmente ligados pelas telecomunicações, e esses fornecem o suporte para o fluxo comunicacional de informação. Rüdiger (2011, p. 76) cita o manual de tecnologia de Friederich Lamprecht de 1787, que afirma que "tecnologia é a ciência fabril que ensina os fundamentos e meios pelos quais os elementos naturais podem se tornar aptos a satisfazer as necessidades humanas". Segundo Tofts (2002, p.3), a tendência de ver o ser humano na informática ou o termo pós-humano inicia-se na década de 40 do século passado. 0 mundo virtual está tão inserido no comportamento de acesso à informação (principalmente em dispositivos móveis) que a própria definição de ciberespaço já não existe mais (HINCHCLIFFE, 2005), pois esse, implica em um espaço fora do sujeito e transcende o computador pessoal. O mundo virtual não pode ser sobreposto ao mundo real, pois isso é o que faz o sentido de ciberespaço ficar obsoleto (apud GLYN, 2005).

Desde então, o mundo virtual não pode ser resumido em suportes, hardware e software nas telecomunicações. No instante em que vemos as interações, sejam elas por comunicação tradicional ou por máquinas, a discussão se torna imprescindível quando nos deparamos com novos comportamentos do uso da informação via tecnologia agora intrínseco à cultura atual.

\section{A informação nos estudos de internet, cibercultura e cultura da informação}

A cultura de informação é a ocorrência das tecnologias em rede, onde o objeto informacional é representado e acessado de variadas maneiras. Entender a contemporânea cultura de informação como consequência dos estudos de cibernética é uma das formas de olhar a informação em seu aspecto social agora sob a ótica científica, ao identificar o ser humano como o centro dos sistemas tecnológicos de informação. Do ponto de vista científico, a cultura de informação é observada pelos Estudos de Cibercultura e Estudos de Internet que estão sendo moldados quanto às teorias e aplicações metodológicas, como um reflexo das discussões acadêmicas em muitas áreas do conhecimento que se preocupam com os desdobramentos da tecnologia em suas estruturas tradicionais.

Apesar de não ser estimada como uma área científica (JONES, 2006), a área "Estudos de Cibercultura" se consolida como a prática acadêmica que observa o movimento cultural com as tecnologias, que é próprio da atualidade. Possuem uma estrutura teórica de investigação que vem se consolidando nos últimos anos devido ao crescimento do interesse acadêmico por Estudos de Internet (SILVER, 2006).

A Ciência da Informação por sua vez, observa os processos pertinentes ao fluxo de informação, mas ainda não prioriza enfaticamente as práticas culturais da tecnologia. Nesse sentido, a Ciência da Informação talvez não tenha observado o movimento cibercultural de forma mais empírica, fato observado por Svensson (2010) em um contexto específico:

A Biblioteconomia e a Ciência da Informação, provavelmente não incluem áreas de pesquisa como estudos críticos da cibercultura, que predominantemente se concentram em cultura digital e construção cultural da tecnologia da informação como objeto de estudo. 
Assim, visualiza-se a colaboração da cibercultura para a Ciência da Informação a partir de suas discussões e abordagens acerca de usos de tecnologias de informação, com a perspectiva de apreender teoricamente aspectos culturais da informação em um horizonte tecnológico.

Silver (2006, p. 55) considera os Estudos de Cibercultura similar aos Estudos de Internet, bem como os temas relacionados: cultura digital, sociedade da informação e novas mídias. Ainda assim, é possível encontrar temas de estudos parecidos, como observado por Silver: trabalhos cooperativos por computador, teoria de hyper e cybertexto, interação humano-computador. Felinto (2011) sugere a extinção da palavra cibercultura para Estudo de Mídia, como feito por Manovich (apud MACEK, 2005) ao considerar posteriormente a cultura de informação como um estudo paralelo aos estudos de mídia, ou mídia visual.

Macek em 2005, por exemplo, havia sistematizado os conceitos relacionados à tecnologia, cultura e a própria cibercultura em quatro categorias: utópico, informacional, antropológico e epistemológico, como descrito no quadro a seguir:

\section{Conceito Utópico} Conceito Informacional Conceito Antropológico Conceito Epistemológico

\begin{tabular}{|c|c|c|c|}
\hline $\begin{array}{l}\text { Como uma forma de } \\
\text { sociedade utópica } \\
\text { alterada pelas } \\
\text { tecnologias de } \\
\text { informação previstas. } \\
\text { (futurologismo) }\end{array}$ & $\begin{array}{l}\text { Como códigos } \\
\text { (símbolos) culturais da } \\
\text { sociedade da informação } \\
\text { - Analítica, parcialmente } \\
\text { prevista }\end{array}$ & $\begin{array}{l}\text { Como práticas culturais e } \\
\text { estilos de vida relacionadas } \\
\text { com as tecnologias de } \\
\text { informação - Analítico, } \\
\text { orientado para o estado atual e } \\
\text { à história }\end{array}$ & $\begin{array}{l}\text { Como termo para } \\
\text { reflexos sócio- } \\
\text { antropológicos de } \\
\text { novas mídias. }\end{array}$ \\
\hline
\end{tabular}

Quadro 1 - Conceitos relacionados à tecnologia, cultura e cibercultura Fonte: Macek (2005)

Um dos teóricos mais citados desse tema (MACEK, 2005), Pierre Levy, define classicamente o conceito de cibercultura como: “(...) o conjunto de técnicas, práticas, atitudes, de modos de pensamento que se desenvolvem com o crescimento do ciberespaço, definido por meio de comunicação que surge da interconexão mundial dos computadores" (LEVY, 1999, p.17). Mais de 15 anos após essa definição, Pierre Levy desenvolve análises sobre o conceito de 'cultura de comunicação de rede', ou 'Infoesfera', enfatizando a característica dos formatos de dados disponíveis na rede mundial de computadores, (música, voz, imagens, textos, programas, etc.). A sua definição está concentrada principalmente na visão comportamental e social. Nesse caso, não é a definição de tecnologia propriamente, e sim a influência exercida pelas técnicas nas pessoas, e mais profundamente ainda, o "universo oceânico de informações", pois para Levy, a cultura cibernética não é necessariamente o meio de comunicação, e sim o que está inserido nele e como ele está ofertado e manipulado.

Enquanto existe a discussão sobre a informação como insumo de principal interesse social e industrial, há a necessidade de falar sobre o espaço onde a comunicação ocorre, ou espaço virtual, o que em linhas gerais seria o ambiente onde acontecem as manifestações sócio-culturais suscitadas pelas tecnologias de informação e comunicação. Pode ser considerado, segundo Bell (2007) como um "universo paralelo" ou "espaço metal comum", ou ainda um "espaço puro de informação". Portanto não há uma demarcação física específica para espaço, o que é criticamente relatado por Powers (2012) como uma sala sem porta de entrada e saída, e que, mesmo que se queira, não é possível sair. Essa espaço, portanto, muda a natureza da informação, que "é impessoal e imperceptível, um conhecimento despojado de seu contexto para ser transformado em dados digitais, que é um preço a ser pago para fazer de informações uma moeda livremente convertível" (MACEK, op. cit). Tal argumento ressalta que o ambiente cibernético, que ora se coloca como o principal provedor de informação, está inserido como motivador para que as mudanças ressaltadas por Levy, no seu tradicional estudo de cibercultura, sejam parte da observação dos pesquisadores de tecnologias e seus variados reflexos.

Segundo Fragoso, Recuero e Amaral (2012), pesquisadores de muitas áreas do conhecimento tem se voltado para pesquisas na área de internet, por preocupações variadas, como "se libertar dos grilhões das práticas disciplinares tradicionais" (MARKHAM apud FRAGOSO, 2012, p. 28). A própria obra das autoras intitulada Métodos de pesquisa para internet apoia os campos científicos que relacionam as suas áreas com esses novos 
estudos, o que parece ser uma evolução para a área de Estudos da Cultura da Informação, onde não é possível discutir sobre sua disciplinaridade, mas a reconhece como uma manifestação acadêmica.

Para as autoras, a associação entre Estudos de Cibercultura e Estudos de Internet, por exemplo é comum nas Ciências Humanas e Ciências Sociais Aplicadas, em especial no Brasil (2012, p. 31). Ao discutir as duas áreas, Fragoso, Recuero e Amaral observam diferenças (2010, p. 65):

Estudos de Cibercultura e Estudos de Internet e Novas Mídias não são sinônimos, uma vez que cibercultura tomaria um espectro mais amplo de análise, incluindo outros tipos de tecnologia e discursos históricos, sociais, estéticos, etc.

Verrifica-se na prática da manifestação desses estudos, a Associação para Pesquisa de Internet, a Association of Internet Researches (AOIR) que foi fundada em 1999, como resultado da conferencia The World Wide Web and Contemporary Cultural Theory: Metaphor, Magic \& Power, que aconteceu na Universidade de Drake em 1998 (HERMAN, SWISS, 2000). A organização se instituiu como o principal centro de pesquisa internacional sobre Estudos de Internet, cujos objetivos são:

- Fornecer uma organização interdisciplinar e interprofissional para a promoção da pesquisa acadêmica e crítica sobre os aspectos sociais, culturais, políticos, econômicos e estéticos da Internet.

- Organizar e patrocinar conferências regulares para fornecer um fórum para aqueles envolvidos em pesquisa na Internet podem se encontrar e trocar informações sobre o seu trabalho.

- Patrocinar e divulgar informações sobre a pesquisa na Internet através da publicação de um site sobre a World Wide Web e através de outras publicações.

- Favorecer o reconhecimento de estudos Internet como uma área de pesquisa acadêmica, desenvolvimento de currículo e ensino.

- Estabelecer conexões entre a comunidade acadêmica, as indústrias e a Internet

- Incentivar o desenvolvimento de pesquisa e estudo sistemático em temas e áreas de Internet e fenômenos relacionados à Internet. (AOIR, 2015)

Em sua primeira conferência, ocorrida no ano 2000, o objetivo foi a demonstração dos Estudos de Internet como um campo interdisciplinar de pesquisa. A conferência de 2016 tem como tópicos para chamada de trabalho temas como "identificação cultura e mídia; códigos e práticas da cultura de internet, big data e análise preditiva, dispositivos conectados e internet das coisas", e ainda um tema muito relevante para a Ciência da Informação: "filosofia da informação e do conhecimento".

Sobre essa perspectiva, é interessante notar que a discussão sobre a informação, que além de estar em todos os temas, também aparece no campo da filosofia, e isso implica que há uma preocupação em afirmar a informação como algo que transcende a tecnologia na internet.

Ao questionar sobre a natureza da informação, Floridi (2002) aponta esta como uma questão oficial para a nova área de investigação filosófica. Em outro artigo, Floridi (2004) define como objetos da filosofia da informação as dinâmicas tecnológicas - a computação-, o fluxo informacional, seu uso e suas ciências. Ilharco (2004), por sua vez afirma que o problema da informação está intimamente ligado à expansão da sociedade da informação. Por isso destaca-se a importância do fenômeno informacional para a sociedade, as características da informação e da comunicação nas tecnologias, e ainda as ameaças e modificações ao comportamento humano na sua relação com as tecnologias e a própria informação.

A cultura de informação como um campo científico pode ser possível no âmbito interdisciplinar por estudar não só a própria estrutura da tecnologia, mas os comportamentos que se manifestam nesse espaço, pois é possível que a cultura global seja extremamente representada na tecnologia de informação, mesmo que muitos ainda 
não tenham acesso. Quando a cultura é definida como uma manifestação de um povo, em vários aspectos (LARAIA, 2009), o contexto da comunicação em rede é como uma parte da Sociedade da Informação, ou além dela. As novas investigações científicas sobre as máquinas e os seres humanos que começaram com a Cibernética e com o sonho de Alice Hilton no século passado, hoje se tornaram uma realidade, que inclusive transpassa a ciência. Cabe às áreas do conhecimento científico acompanharem essas transformações, desde as áreas da saúde e humanidades até as sociais aplicadas, em especial a Ciência da Informação.

\section{Conclusão}

Na relação entre as tecnologias e sociedade, há de se considerar que o homem sempre quis que seu trabalho fosse facilitado ou automatizado, e isso se justifica pelos avanços das engenharias. Quanto ao uso das tecnologias mais inovadoras, desde aplicativos à internet das coisas, o comportamento nem sempre foi de adaptação, permanecendo atualmente variadas formas de acesso, habilidades e intenções. Não há como distinguir onde há e onde não há tecnologias em processos humanos, por isso, talvez seja possível identificar tantas discussões sobre isso nos meios de comunicação. E com isso nos vemos diante da cultura imersa em tecnologias de informação ou ainda, uma cultura buscando incessantemente por informações em tempo real. Esse movimento sob o viés da ciência direciona-se a um objeto comum em todas as abordagens: a informação. Nesse aspecto, a contribuição da Ciência da Informação é histórica e avança em diversas frentes de pesquisa, e se talvez essa contribuição não seja visível, é porque simplesmente todas as intervenções se fazem de maneira multidisciplinar. Isso se justifica pelo fato de que a transmissão de informação e conhecimento está potencializada e acelerada pelas tecnologias de comunicação. Por esse motivo, alguns aspectos da própria cultura de informação podem estar implícitos nas pesquisas de muitas ciências, em especial na Ciência da Informação, principalmente quando existe a associação das tecnologias de informação com os objetivos das pesquisas.

A entrada da Ciência da Informação no rol das áreas cientificas que se preocupam com a cultura da tecnologia, poderia não só colaborar com esses estudos, como trazer colaborações interdisciplinares e ainda dar maior visibilidade para a área da Ciência da Informação.

Assim, consideramos que a Ciência da Informação possa colaborar em conjunto com as outras ciências, com uma visão mais humana, prática e organizacional ao otimizar os processos do fluxo de informação, e se ocupando da comunicação do conhecimento para a inteligência. E todas as ciências que se propõe a estudar a cultura da informação podem fortalecer a qualidade das interações entre homem e a máquina, como foi projetado pela ciência da cibernética e por Alice Hilton.

\section{Referências}

AOIR. Association of Internet Researchers. Disponível em < http://aoir.org/>. Acesso em: 02 fev. 2016.

BAUMAN, Z. Modernidade liquida. Rio de Janeiro: Zahar, 2001.

CARR, Nicholas. A geração superficial: o que a internet está fazendo como os nossos cérebros. Tradução de Mônica Gagliotti Fortunato França. Rio de Janeiro: Agir, 2011. Tradução de Mônica Gagliotti Fortunato França.

The glass cage. EUA: WW Borton, 2014.

DECHERT, Charles R. (Coord.). O impacto social da cibernética. Tradução de Adilson Alkimin Cunha. Rio de Janeiro: Bloch, 1970.

EPSTEIN, Isaac (Org.). Cibernética e comunicação. São Paulo: Cultrix, 1973.

FRAGOSO, Suely; RECUERO, Raquel; AMARAL, Adriana. Métodos de pesquisa para internet. Porto Alegre: Sulina, 2012.

FRANK, Helmar G. Cibernética e filosofia. Rio de Janeiro: GB, 1970.

FELINTO, Erick. Sem mapas para esses territórios: a cibercultura como campo do conhecimento. INTERCOM, 30.; 2007. Santos, SP, 2007. p. 1-14. Disponível em: <http://goo.gl/GVCiV>. Acesso em 02 fev. 2016.

Cibercultura: ascensão e declínio de uma palavra quase mágica. E-Compós, Brasília, v. 14, p. 1-14, 2011. Disponíve em: <http://www.compos.org.br/seer/index.php/e-compos/article/view/548/511>. Acesso em 02 fev. 2016. 
FLORIDI, Luciano. What is the Philosophy of Information? Metaphilosophy, Vol.33, 1/2, 2002

. Open Problems in the Philosophy of Information. Metaphilosophy, Vol. 35, n.3, Abril de 2004

GLYN, Ruairi. Karl Schroeder, cyberespace, RIP. Disponivel em:< http://www.interactivearchitecture.org/karl-schroedercyberspace-rip.html >. Acesso em 02 fev. 2016.

HERMAN, Andrew; SWISS, Thomas. The world wide web and contemporary cultural theory: magic, metaphor, power. USA: Routledge, 2000.

HILTON, Alice Mary. An ethos for the age of cyberculture. In:Spring joint computer conference, New York, p. 139-153, 1964. Disponivel em:< http://dl.acm.org/ft gateway.cfm? id=1464136\&type=pdf\&CFID=95289528\&CFTOKEN=43067794>. Acesso em 02 fev. 2016.

HINCHCLIFFE, Dion. Will web 2.0 kill cyberspace? Disponível em:< http://laszlo.sys-con.com/node/141534>. Acesso em 02 fev. 2016.

ILHARCO, Fernando. Filosofia da informação: alguns problemas fundadores. II Congresso Ibérico de Ciências da Comunicação. Sessão Temática: Teorias da Comunicação. Disponível em: < http://www.ucp.pt/site/resources/documents/FCH/F\%20llharco/fernando ilharco filosofia informacao problemas fundadores.p df >. Acesso em 02 fev. 2016.

JOHNSON, Clay A. A dieta da informação: uma defesa do consumo consciente. São Paulo: Novatec,2012.

JONES, Steve. Foreword dreams of fields: possible trajectories of internet studies. In: SILVER, David; MASSANARI, Adrienne (Orgs.). Critical cyberculture studies. New York: New York University, 2006.

LARAIA, Roque de Barros. Cultura: um conceito antropológico. 23. ed. Rio de Janeiro: Jorge Zahar, 2009.

LEMOS, André; LÉVY, Pierre. O futuro da internet: em direção a uma ciberdemocracia planetária. São Paulo: 2010.

LÉVY, Pierre. Cibercultura. São Paulo : Ed. 34, 1999.

LINS, Greyciane. Colaborações dos estudos de cibercultura para a ciência da informação. 2013. 17 f. Tese (Doutorado em Ciência da Informação). UnB, Brasília, 2012.

MACEK, Jakub. Defining cyberculture. jul 2005.Disponível em <http://macek.czechian.net/defining cyberculture.htm>. Acesso em 02 fev. 2016.

POWERS, Willian. O blackberry de Hamlet: filosofia prática para viver bem na era digital. São Paulo: Alaúde, 2012.

RÜDIGUER, Francisco. As teorias da cibercultura: perspectivas, questões e autores. São Paulo: Salinas, 2011.

SHANNON, C. E. A mathematical theory of communication. The Bell System Technical Journal, vol. 27, n. 3-4, p. 379-423 and 623-656, July and Oct 1948. Disponível em:< http://worrydream.com/refs/Shannon\%20$\% 20$ A $\% 20$ Mathematical\%20Theory\%20of\%20Communication.pdf >. Acesso em 02 fev. 2016.

SILVER, David; MASSANARI, Adrienne (Orgs.). Critical cyberculture studies. New York: New York University, 2006.

TOFTS, Darren; JONSON, Annemarie; CAVALLARO, Alessio. Prefiguring cyberculture: an intellectual history. Cambridge: The MIT Press, 2002

WIENER, Norbert. Cibernética e sociedade: o uso humano de seres humanos. 15. ed. São Paulo: Cultrix, 2006. 


\section{Dados da autora}

Greyciane Souza Lins

Doutora em Ciência da Informação. Possui graduação em Biblioteconomia pela Universidade de Brasília (2003) e mestrado em Ciência da Informação pela Universidade de Brasília (2007). Tem experiência na área de Ciência da Informação, com ênfase em Processos de Disseminação da Informação, atuando principalmente nos seguintes temas: organização e recuperação da informação, sistemas de informação, educação e tecnologia, comunicação, cibercultura, competência informacional e tecnológica.

greycilins@gmail.com

Recebido - Received: 2015-11-16

Aceitado - Accepted: 2016-02-02

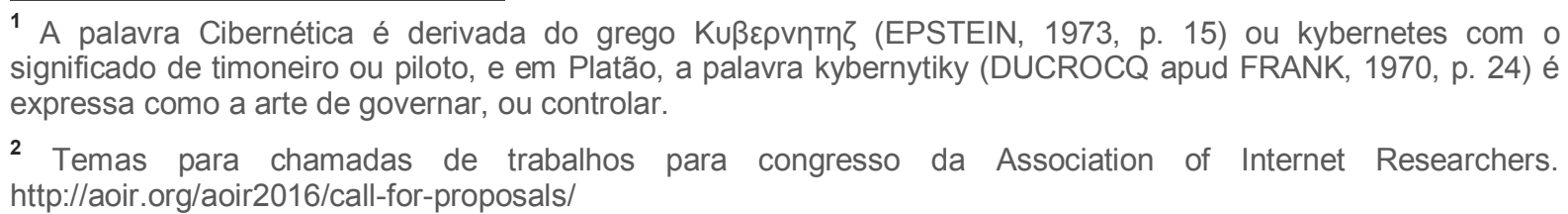

\section{(c) $)$ EY}

This work is licensed under a Creative Commons Attribution 4.0

United States License.

\section{UILIS D}

This journal is published by the University Library System of the University of Pittsburgh as part of its D-Scribe Digital Publishing Program and is cosponsored by the University of Pittsburgh Press. 